\title{
Fungal Endophytes of Populus trichocarpa Alter Host Phenotype, Gene Expression, and Rhizobiome Composition
}

\author{
Hui-Ling Liao, ${ }^{1,+}$ Gregory Bonito, ${ }^{2}$ J. Alejandro Rojas, ${ }^{3,4}$ Khalid Hameed, ${ }^{3}$ Steven $\mathrm{Wu},{ }^{5}$ \\ Christopher W. Schadt, ${ }^{6}$ Jessy Labbé, ${ }^{6}$ Gerald A. Tuskan, ${ }^{6}$ Francis Martin, ${ }^{7}$ Igor V. Grigoriev, ${ }^{8}$ and \\ Rytas Vilgalys ${ }^{3}$ \\ ${ }^{1}$ North Florida Research and Education Center, University of Florida, 155 Research Road, Quincy, FL 32351, U.S.A. \\ ${ }^{2}$ Department of Plant, Soil and Microbial Sciences, Michigan State University, East Lansing, MI 48824, U.S.A. \\ ${ }^{3}$ Department of Biology, Duke University, Durham, NC, U.S.A. \\ ${ }^{4}$ Plant Pathology Department, University of Arkansas, 211 PTSC-Fayetteville, AR 72701, U.S.A. \\ ${ }^{5}$ Independent Researcher, Davis, CA, U.S.A. \\ ${ }^{6}$ Oak Ridge National Laboratory, 1 Bethel Valley Road, Oak Ridge, TN 37830, U.S.A. \\ ${ }^{7}$ INRA, UMR 1136 INRA-University of Lorraine, Interactions Arbres/Microorganismes, Laboratory of Excellence ARBRE, INRA- \\ Nancy, 54280, Champenoux, France \\ ${ }^{8}$ U.S. Department of Energy Joint Genome Institute and Department of Plant and Microbial Biology, University of California \\ Berkeley, 2800 Mitchell Drive, Walnut Creek, CA 94598, U.S.A.
}

Accepted 28 January 2019.

\begin{abstract}
Mortierella and Ilyonectria genera include common species of soil fungi that are frequently detected as root endophytes in many plants, including Populus spp. However, the ecological roles of these and other endophytic fungi with respect to plant growth and function are still not well understood. The functional ecology of two key taxa from the $P$. trichocarpa rhizobiome, M. elongata PMI93 and I. europaea PMI82, was studied by coupling forest soil bioassays with environmental metatranscriptomics. Using soil bioassay experiments amended with fungal inoculants, $M$. elongata was observed to promote the growth of $P$. trichocarpa. This response was cultivar independent. In contrast, I. europaea had no visible effect on $P$. trichocarpa growth. Metatranscriptomic studies revealed
\end{abstract}

${ }^{\dagger}$ Corresponding author: H.-L. Liao; sunny.liao@ufl.edu

Funding: This work was supported by the Plant-Microbe Interfaces program at the Oak Ridge National Laboratory (ORNL) sponsored by the Office of Biological and Environmental Research at the United States Department of Energy (DOE) Office of Science. ORNL is managed by UTBattelle, LLC, under contract DE-AC05-00OR22725 for the DOE. The work conducted by the DOE Joint Genome Institute, a DOE Office of Science User Facility, is supported by the Office of Science of the United States Department of Energy under contract DE-AC02-05CH11231. Support was also provided by the NSF Zygolife (NSF-DEB1441715), the United States National Science Foundation (NSF) (DEB 1737898 to G. Bonito), the Laboratory of Excellence Advanced Research on the Biology of Tree and Forest Ecosystems (ARBRE) (ANR-11-LABX 0002 01 to F. Martin), JGI Community sequencing program project (CSP570, DE-AC02-05CH11231), and National Science Foundation (concept ID 10.13039/100000001; grant number DEB 1737898).

*The $\boldsymbol{e}$-Xtra logo stands for "electronic extra" and indicates that eleven supplementary figures, two supplementary tables, supplementary text, and eight supplementary datasets are published online.

The author(s) declare there is no conflict of interest.

() 2019 The American Phytopathological Society that these fungi impacted rhizophytic and endophytic activities in P. trichocarpa and induced shifts in soil and root microbial communities. Differential expression of core genes in $P$. trichocarpa roots was observed in response to both fungal species. Expression of $\boldsymbol{P}$. trichocarpa genes for lipid signaling and nutrient uptake were upregulated, and expression of genes associated with gibberellin signaling were altered in plants inoculated with M. elongata, but not I. europaea. Upregulation of genes for growth promotion, downregulation of genes for several leucine-rich repeat receptor kinases, and alteration of expression of genes associated with plant defense responses (e.g., jasmonic acid, salicylic acid, and ethylene signal pathways) also suggest that $M$. elongata manipulates plant defenses while promoting plant growth.

Soils of Populus spp. and forest trees harbor a high diversity of rhizospheric fungi with diverse ecological functions, including mycorrhizal fungi, endophytes, saprophytes, and pathogens (Bonito et al. 2016). In particular, Populus spp. are associated with a high diversity of root endophytes that play key roles in rhizosphere function and plant fitness (Cregger et al. 2018; Shakya et al. 2013). While several Populus spp.-ectomycorrhizal interactions have been reported (Baum and Makeschin 2000; Bois et al. 2005; Gottel et al. 2011; Guevara et al. 2013; Lodge 1989; Martin et al. 2016; Podila et al. 2009), the mechanisms involved in Populus spp.--endophyte interactions that affect plant growth and fitness remain unexplored.

Recent studies have identified Mortierella spp. as part of the core Populus spp. microbiome (Bonito et al. 2014; Gottel et al. 2011; Shakya et al. 2013; Uehling et al. 2017). Mortierella spp. belongs to Mucoromycota, an early diverging phylum of fungi, that is comprised of Glomeromycotina (arbuscular mycorrhizal fungi $[\mathrm{AMF}]$ ), Mortierellomycotina, and Mucoromycotina (Bidartondo et al. 2011; Spatafora et al. 2016; Strullu-Derrien et al. 2018). Most Mortierella spp. are considered to be soil saprophytes; however, they are also frequently isolated as 
endophytes from surface-sterilized healthy root tissue of Populus and other plant species (Bonito et al. 2016). Beneficial interactions between Mortierella spp. and plants are known to exist, but there are few functional and mechanistic studies on plant-Mortierella spp. interactions. A recent study showed that M. hyalina enhanced the aboveground biomass of Arabidopsis and activated host $\mathrm{Ca}^{2+}$ signaling to suppress immune responses (Johnson et al. 2019). Another study showed the ability of endophytic $M$. alpina to enhance the stress tolerance in host plants as a root endophyte via biosynthesis of the tetraterpenoid-derived phytohormones in planta, including apocarotenoid (Wani et al. 2017). Genome analysis and carbon utilization assays suggest that $M$. elongata metabolism is largely based on simple carbon utilization (e.g., D-glucose, Dtrehalose, and D-mannose) and that its metabolism is enriched in lipids and polyunsaturated fatty acid anabolism (Uehling et al. 2017). Based on their enzyme profile, Mortierella spp. can acquire organic nitrogen through chitinolytic activities (Uehling et al. 2017; Vadivelan and Venkateswaran 2014) by utilizing the chitin monomer $\mathrm{N}$-acetyl glucosamine as a nitrogen and carbon source.

Ilyonectria spp. are another common group of ubiquitous rhizosphere fungi whose function as endophytes is poorly understood. Ilyonectria spp. belongs to the family Nectriaceae (Hypocreales, Sordariomyceta, Ascomycota); however, the taxonomy status of Ilyonectria and other related genera is still in flux (Chaverri et al. 2011). For instance, molecular systematic studies have revealed a high degree of cryptic speciation within the Ilyonectria spp. complex (Cabral et al. 2012).

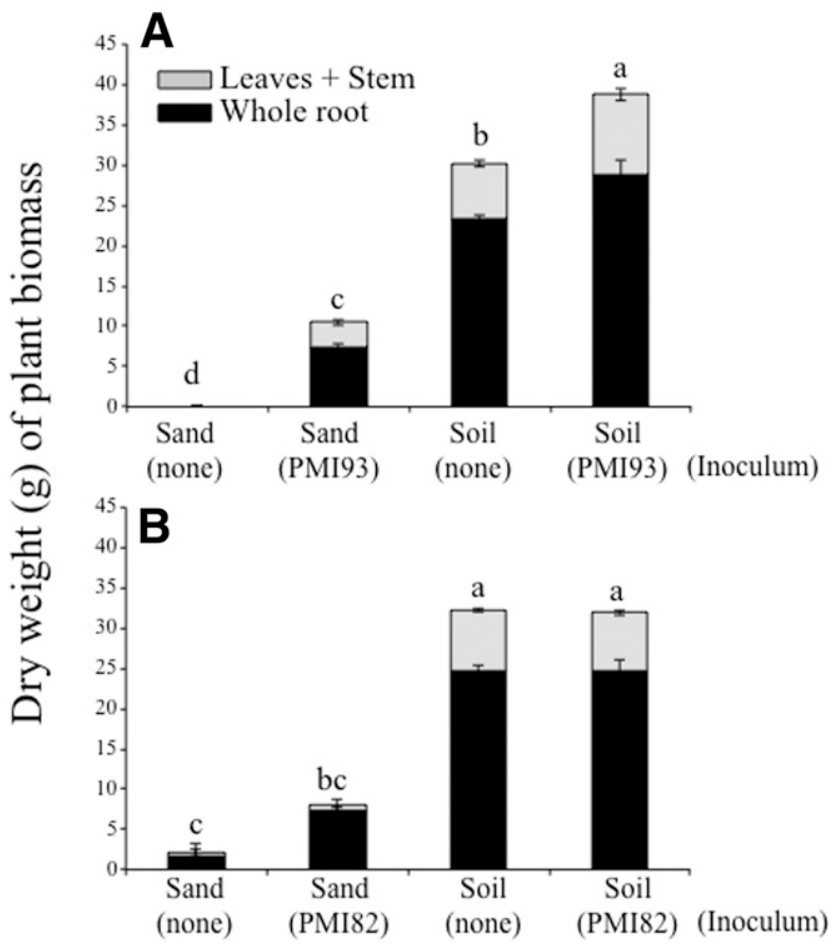

Fig. 1. Plant dry weight (Populus trichocarpa BESC4) in response to inoculation with A, Mortierella elongata (PMI93) and B, Ilyonectria europaea (PMI82). Two soil treatments, sterile sand and $30 \%$ natural soil (wt/wt) $/ 70 \%$ sterile sand (wt/wt) collected from $P$. trichocarpa site (NC1 in the United States), were used. The data were collected 1-year postinoculation. Error bars indicate standard deviation of data for aboveground tissues (error bars above) and root tissues (error bar below) $(n=4)$. The Tukey test was used to test the significance of the whole plant biomass across the combinations $(P \leq 0.05 ; n=4)$. Means marked with the same letters were not significantly different.
Ilyonectria spp. are commonly isolated from rhizosphere soils and as endophytes from surface-sterilized healthy roots from a wide range of woody and herbaceous plants, including Populus spp. (Cui et al. 2015; Kwaśna et al. 2016). Ilyonectria spp. are generally assumed to be commensals or weak plant pathogens, since some species are associated with diseases of certain plant hosts, including root rot in grapes (Cabral et al. 2012; Hersh et al. 2012) and ginseng (Farh et al. 2018). However, as with Mortierella spp., the ecological function of most Ilyonectria spp. is unknown.

We hypothesize signaling between Populus spp. and other nonmycorrhizal fungal root endophytes can occur in a community context and is bidirectional, such that each symbiont impacts the transcriptional regulation of its partner. However, we predict that $P$. trichocarpa uses different strategies to interact with $M$. elongata compared with I. europaea given that these fungal taxa are separated by a large phylogenetic distance and are assumed to represent opposite ends of the "pathogen-mutualist" ecological spectrum. We carried out bioassay experiments with P. trichocarpa to test our hypotheses that M. elongata (PMI93) and I. europaea (PMI82) elicit different molecular responses from their native host $P$. trichocarpa. We used RNA-Seq to investigate the functional activities of these fungal generalists (PMI93 and PMI82) in the rhizosphere and to identify the key plant genes that are responsive to fungal inoculation. This study highlights how different functional groups of endophytic fungi interact with a single host plant, $P$. trichocarpa, and provides new insights into the relationships between species coexistence, plant fitness, and ecosystem functions.

\section{RESULTS}

\section{M. elongata (PMI93) promotes plant growth.}

To test whether endophytic fungal taxa belonging to the core Populus spp. rhizobiome contribute to plant fitness, bioassay experiments were used to examine the response of $P$. trichocarpa to inoculation with two fungi isolated from $P$. trichocarpa roots, M. elongata (PMI93) and I. europaea (PMI82) (Bonito et al. 2016). $P$. trichocarpa cuttings were grown in a background of soils collected from $P$. trichocarpa growth sites in North Carolina and inoculated with $M$. elongata (PMI93) or I. europaea (PMI82), respectively. M. elongata (PMI93) enhanced wholeplant dry weight $(30 \% ; P \leq 0.05)$ and leaf expansion $(P \leq 0.05)$. Additionally an increased amount of chloroplasts was observed in P. trichocarpa (Fig. 1; Supplementary Fig. S1). Particularly, M. elongata (PMI93) enhanced the dry weight in P. trichocarpa roots more than it did in aboveground organs (Fig. 1). When $P$. trichocarpa was grown in both soil and sand, growth of aboveground organs triggered by $M$. elongata (PMI93) was not plant genotype-dependent. In contrast, I. europaea (PMI82) promoted $P$. trichocarpa growth in sterilized sand, but otherwise had no effect on plant growth (Fig. 1B). The response of $P$. trichocarpa to I. europaea was investigated only on a single genotype, BESC4 (Fig. 1B), so a positive or negative response with other genotypes cannot be excluded.

\section{Fungi as biotic factors that influence the composition of fungal communities in $P$. trichocarpa roots and soils.}

RNA-Seq data indicate that there were populations of M. elongata and I. europaea in the soils used for these bioassays (Supplementary Dataset S1; Fig. 2); however, inoculation resulted in a larger population of the target taxa and a higher relative abundance of target mRNA recovered (Fig. 2A). Inoculation with $M$. elongata (PMI93) increased the target fungus only in soil samples, whereas inoculation with I. europaea (PMI82) resulted in increases in both root and soil samples (Fig. 2A). M. elongata (PMI93) inoculation resulted in an 
increase in the relative abundance of $I$. europaea mRNA in the roots and soil (Fig. 2B). I. europaea (PMI82) inoculation resulted in an increase in the relative abundance of $M$. elongata mRNA in the soil (Fig. 2B).

To explore the interactive effects of M. elongata (PMI93) and I. europaea (PMI82) on the fungal community in P. trichocarpa roots and soils, fungal large subunit (LSU) ribosomal RNA reads (corresponding to divergent domains D1 and D2) were extracted from the RNA-Seq data and used to identify fungal community composition in soils and roots as described by Liao et al. (2014). Species-rich communities of root-associated fungi were detected in individual $P$. trichocarpa roots and rhizosphere soils (Fig. 3). A high diversity of fungi across different ecological guilds, from mutualists to pathogens, was present in all samples. A block effect was observed (Fig. 3), whereby the microbial community structure of noninoculated $M$. elongata (PMI93) samples was more similar to inoculated $M$. elongata (PMI93) samples and the microbial community structure of noninoculated I. europaea (PMI82) samples was more similar to inoculated I. europaea (PMI82) samples, which is likely explained by the fact that two different soils (harboring different microbial communities) were used for the two separate experiments. Therefore, the two experiments were analyzed independently. Further, higher variability of soil and root fungal community structures was found in the I. europaea (PMI82) experimental samples compared with M. elongata (PMI93) experimental samples, regardless of the addition of fungal inoculum (Supplementary Fig. S3; Supplementary Table S2). More than $90 \%$ of the detected fungi were root-associated fungi, including $\mathrm{AMF}$ and ectomycorrhizal fungi (EMF), endomycorrhizal fungi, and fungal endophytes. It is not known whether the physiological conditions of $P$. trichocarpa cuttings used for the individual replicates contributed to the variability of fungal microbiomes. The physiological conditions of plants were not examined other than the measurement of plant biomass (Fig. 1). In addition, inoculation with either M. elongata (PMI93) or I. europaea (PMI82) resulted in a shift in the composition of the fungal soil community compared with noninoculated plants grown in the same soils $(P \leq 0.05)$. AMF transcriptome activity changed significantly in the roots and soils inoculated with I. europaea (PMI82), but not with $M$. elongata (PMI93) $(P \leq 0.05)$. M. elongata (PMI93) inoculation reduced some AMF taxa in root tissues, including those of Glomeromycota, Septoglomus, and Scutellospora (Fig. 3). Soil inoculation with M. elongata (PMI93) resulted in increased transcriptome activity by EMF, an effect that has also been observed for other fungi in the Nectriaceae (Swett and Gordon 2017). The transcriptome composition of endophytes in the soils was shifted in response to both M. elongata (PMI93) and I. europaea (PMI82) inoculation. Inoculation with M. elongata (PMI93) resulted in enrichment of certain fungal endophytes (Gibberella, Bionectria, Neonectria, Neocosmospora, Nectria, and Trichoderma) in both root and soil systems (Fig. 3). I. europaea (PMI82) inoculation resulted in the enrichment of the fungal pathogens Leptosphaerulina and Didymella (Fig. 3). Finally, inoculation with M. elongata (PMI93) and I. europaea (PMI82) resulted in a shift in the composition of transcribed rRNA of saprotrophs.

\section{Shared and unique responses of $P$. trichocarpa responses to $M$. elongata (PMI93) and \\ I. europaea (PMI82) inoculations.}

The net transcriptomic activity of $P$. trichocarpa roots with and without fungal inoculation was compared. Approximately 34 million reads were recovered from individual pools of fine roots (around $1 \mathrm{mg}$ ). The average read proportion of expressed genes was $72 \%: 0.1 \%: 28 \%$, (plant/inoculated fungi/other species not mapped to plant hosts or inoculated fungal genomes) for individual samples regardless of whether the samples were inoculated with fungi (Fig. 2). Additional details of the computational pipeline used for data assemblies can be found in Supplementary Figure S2.

Inoculation with M. elongata (PMI93) and I. europaea (PMI82) resulted in a strong molecular plant response (Fig. 4;
A

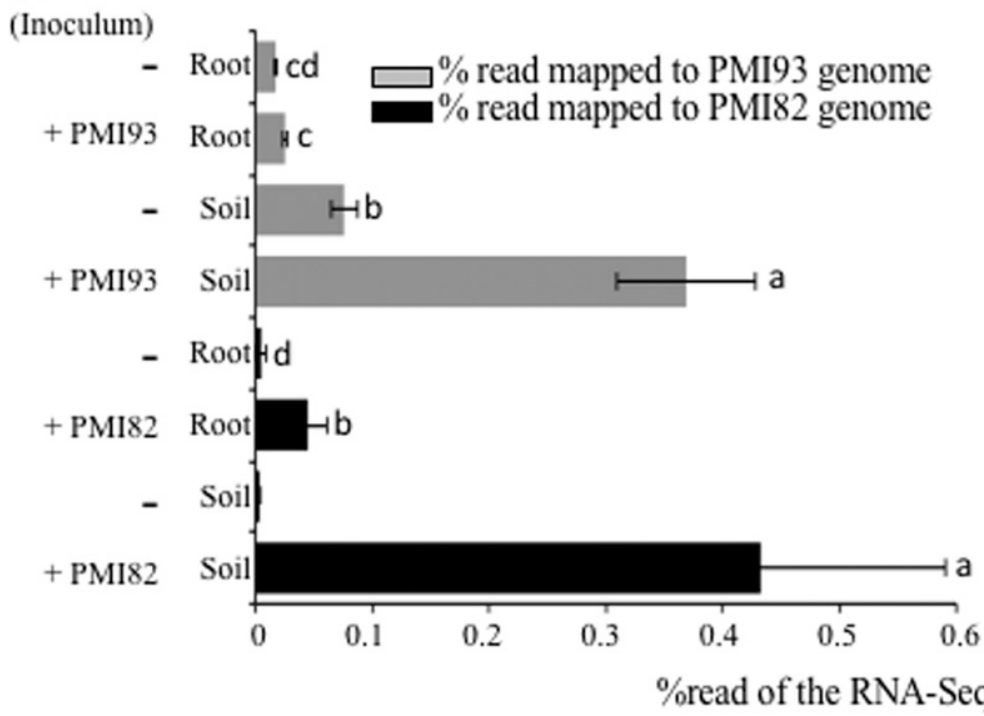

B

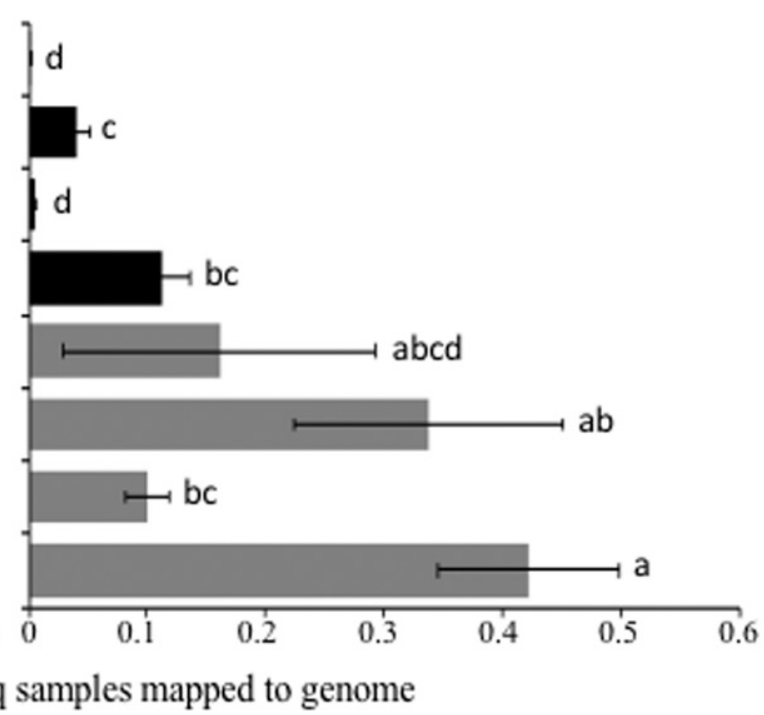

Fig. 2. Mortierella elongata (PMI93) and Ilyonectria europaea (PMI82) are transcriptomically active in forest soil and Populus trichocarpa roots enriched by inoculation. Bars show the percentage of total RNA-Seq reads of root and soil samples ( + and $-=$ with and without inoculum, respectively) mapped to the genome databases of $M$. elongata and I. europaea, respectively. A, Individual sample sets mapped to the genome databases of their inoculum; B, individual sample sets mapped to the genome databases of $I$. europaea or M. elongata, but not inoculum. Error bars indicate standard deviation of the data $(n=4)$. The Tukey test was used to test the significance of the percentage of reads across the combinations in a bar graph $(P \leq 0.05 ; n=4)$. Means marked with the same letters were not significantly different. 
Supplementary Dataset S2). More than 4,497 individual genes of $P$. trichocarpa were significantly altered in response to M. elongata (PMI93) inoculation (false discovery rate [FDR] $\leq 0.05$; greater than or equal to twofold changes; $n=4$ ) (Fig. 4A). In contrast, only 380 genes of $P$. trichocarpa changed their expression patterns in response to inoculation with I. europaea (PMI82) (Fig. 4A). Further, replicate samples of I. europaea (PMI82) appeared to be much more variable than for M. elongata (PMI93) (Figs. 3 and 5). It is likely that the higher variability in gene expression across replicates was the cause of fewer differentially expressed $P$. trichocarpa genes in response to I. europaea (PMI82) inoculation compared with M. elongata (PMI93) inoculation.

The expression patterns of 260 genes changed in $P$. trichocarpa in response to inoculation with both $M$. elongata (PMI93) and I. europaea (PMI82) (shared genes) (Fig. 5; Supplementary Dataset S3), which is indicative of molecular commonalities in how $P$. trichocarpa responds to different species of rhizosphere fungi. Pathways mediating this shared response include carbohydrate metabolism, plant cell wall development, fatty acid or lipid biosynthesis and metabolism, indole-3-acetic acid (IAA) signaling, heat shock, stress response and transport, and intracellular signaling and transcriptional regulation. As part of this common response, a few functional groups, including several plant defense-related genes (18 genes), are significantly up- and downregulated (FDR $\leq 0.05$; greater than or equal to twofold changes; $n=4)$. These include jasmonic acid (JA), ethylene (ET), and abscisic acid
(ABA) biosynthesis and signaling and salicylic acid (SA)leucine-rich repeat (LRR)-mediated signaling. For example, one gene for JA signaling (12-oxophytodienoate reductase) was upregulated (Gene ID: Potri.013G102700), and two genes encoding lipoxygenase were downregulated (Gene ID: Potri.005G032700; Potri.005G032400) (Supplementary Table S1). The NDR1/NIH1-like gene (Gene ID: Potri.017G154000), which has been reported to respond to SA-mediated biotic stress, also was upregulated in response to fungal inoculation (Wu et al. 2012).

Aside from shared genes, 4,237 and 120 P. trichocarpa genes were predicted to respond to $M$. elongata (PMI93) and I. europaea (PMI82), respectively. P. trichocarpa responded more strongly to $M$. elongata (PMI93) compared with I. europaea (PMI82) at physiological and molecular levels. Thus, further analyses focused specifically on $P$. trichocarpa responses to $M$. elongata (PMI93). The majority of the functional groups of these unshared genes were involved in transmembrane functions (32\% of total unshared genes), extracellular functions $(7 \%)$, and transcriptional regulation $(8 \%)$ regardless of soil batch or fungal species used as inoculum (Supplementary Fig. S4). Of 356 plant genes involved in extracellular activities during $P$. trichocarpa-M. elongata (PMI93) interaction, 147 genes (41\% of extracellular proteins) were considered to be plant small secreted proteins (pSSP), comprising up to $8 \%$ of total pSSP $(1,680 \mathrm{pSSP})$ from the P. trichocarpa genome (Tuskan et al. 2006; Yang et al. 2011). Of 147 P. trichocarpa pSSP, 94 were upregulated, and 53 genes
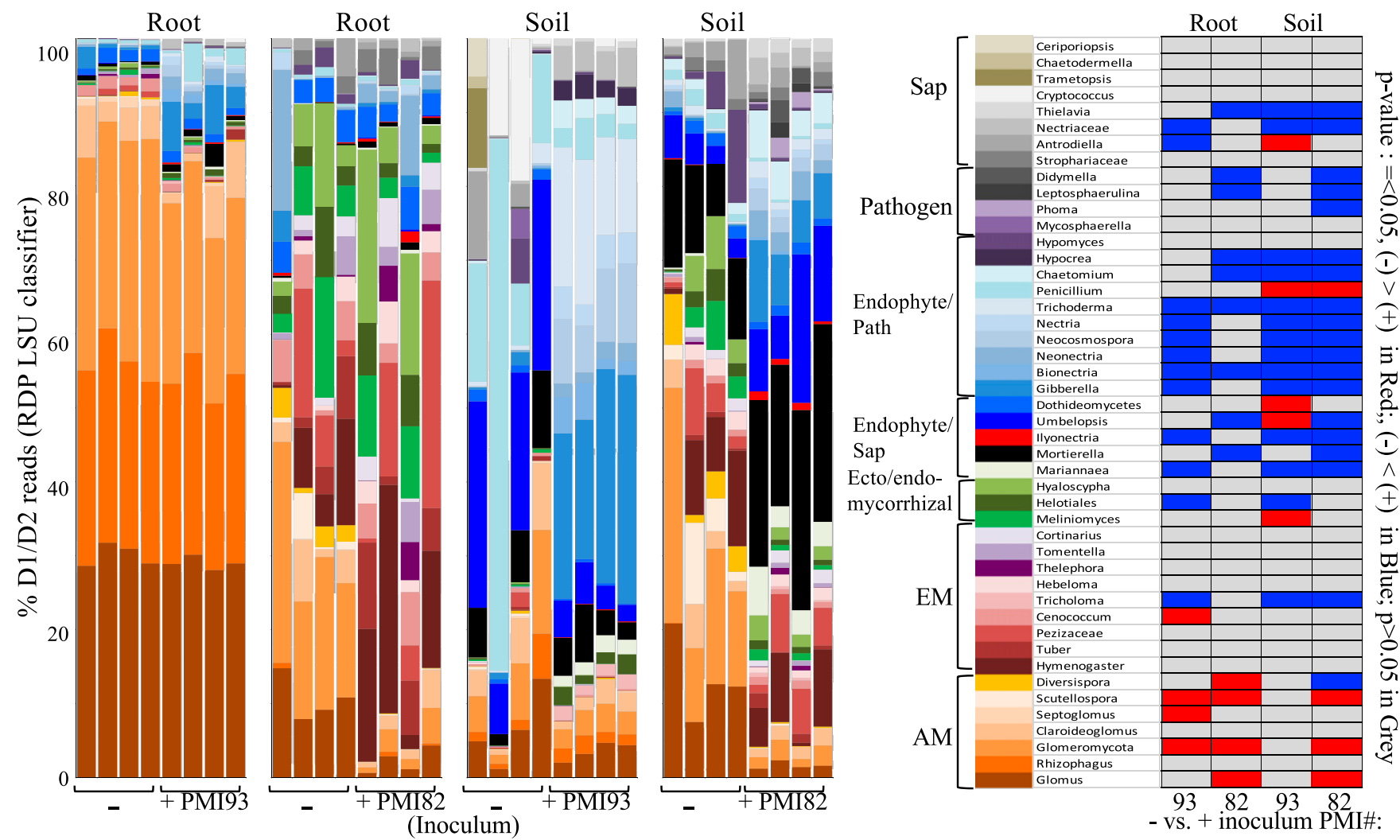

Fig. 3. Taxonomic composition of fungal communities in noninoculated (-) root and soil samples and those inoculated (+) with Mortierella elongata (PMI93) and Ilyonectria europaea (PMI82). Individual bars show the normalized abundance of large subunit ribosomal RNA sequences (LSU D1D2). Biological replicates $(n=4)$ show a consistent taxonomic representation of reads recovered from root and soil samples across treatments. Percentages indicate the relative values of paired reads. The relative values $<1$ appear as 0 (more than $80 \%$ of the relative values $<1$ were singleton representatives, data not shown). Taxa are ordered and coded in colors based on the ecological function of the fungal taxa according to FunGuild (Nguyen et al. 2016). Permutational multivariate analysis of variance (PERMANOVA) was applied to identify the taxa with significant differential abundance associated with Mortierella elongata (PMI93) and Ilyonectria europaea (PMI82) inoculation. In the panel of boxes on the right, the abundance of normalized rRNA reads (using Deseq2) that was significantly increased (blue boxes) or reduced (red boxes) $(P \leq 0.05)$ or not significantly changed (gray boxes) $(P>0.05)$ are shown. 
were downregulated in response to M. elongata (PMI93) (Fig. 6; Supplementary Dataset S4). Genes encoding pSSP involved in plant lipid-transfer proteins (pLTP) and cell wall loosening (expansin) were upregulated, whereas genes encoding pSSP related to cell adhesion and plant defense response were downregulated.

Of 85 plant genes involved in extracellular activities during P. trichocarpa-I. europaea (PMI82) interaction, 15 genes $(18 \%)$ were predicted to be pSSP. One gene encoding an pSSP for pLTP (Gene ID: Potri.013G131500), two genes for protein app1, and three genes for cell wall protein gp1-like were upregulated. Other genes encoding pSSP, including serine protease inhibitor (Kazal-type), clavata3, and plant natriuretic peptide A, were downregulated. None of these 15 genes encodes for cell adhesion and plant defense response. Protein structure analysis was further applied to study the structural architectures of these pSSP groups (Supplementary Fig. S5).

Two distinct pLTP families (family 1 LTP and family 2 LTP) have been biochemically characterized (Yeats and Rose 2008). All 12 P. trichocarpa pLTP identified in response to $M$. elongata (PMI93) shared the structural architecture of a hydrophobic cavity enclosed by four $\alpha$-helices that were folded using four disulfide bounds. The conserved eight-cysteine motif contributed to these four disulfide bounds. The presence of tyrosine-16 and small hydrophobic amino acids (isoleucine, valine, leucine, alanine) directed $P$. trichocarpa $\mathrm{pLTP}$ as the family 1 pLTP. These $P$. trichocarpa pLTP varied in amino acid identity (between 1 and 90\% identity).

$P$. trichocarpa $\mathrm{pSSP}$ associated with defense responses were predominantly downregulated in response to $M$. elongata (PMI93), including three groups-dirigent-like protein, germinlike protein, and PR-thaumatin-associated protein (Fig. 6). Ligand and enzyme activity prediction analysis showed different modes of the plant immune systems, including JA-, ET-, SA-, and ABA-mediated pathways, were suppressed in association with $M$. elongata (PMI93) inoculation. Along this line, although one gene for JA signaling (12-oxophytodienoate; Gene ID: Potri.013G102700) was upregulated in $P$. trichocarpa in response to both $M$. elongata and I. europaea inoculation, other 12-oxophytodienoate reductases (Gene ID: Potri.003G004600 and Potri.003G004200) were conversely downregulated in the root inoculated with $M$. elongata (PMI93). All detected genes for lipoxygenases (Gene ID: Potri.005G032400, Potri.005G032700, Potri.005G032600, Potri.013G022100, and Potri.009G022400) were also downregulated in roots inoculated with $M$. elongata (PMI93). Expression of $23 P$. trichocarpa genes for gibberellin (GA) signaling was altered in plants inoculated with $M$. elongata, but not I. europaea.

We further categorized these genes using different annotation methods, including KEGG mapper (Supplementary Fig. S6), KOG gene groups (JGI annotation) (Supplementary Fig. S7), and ClueGO gene-enrichment analysis (Bindea et al. 2009, 2013) (Supplementary Fig. S8). Results of these analyses indicate that M. elongata (PMI93) inoculation contributed to an upregulation in $P$. trichocarpa pathways involved in fatty acid and glycerolipid biosynthetic processes and metabolism and in oxidative phosphorylation. Conversely, there was a downregulation of genes involved in carotenoid biosynthesis and ET, JA, and SA signaling $(P \leq 0.05$; greater than or equal to twofold changes; $n=4)$. The most abundant differentially expressed genes appeared to be mostly involved in signaling (Figs. 5 and 6), including receptor kinases and transcription factors. The majority of receptor kinases were downregulated in response to M. elongata (PMI93), including 61 genes for LRR-receptor kinases (LRR-RK). ClueGo gene-enrichment analysis also showed that inoculation with $M$. elongata (PMI93) enhanced the activities of fatty acid biosynthesis, thiolester hydrolase, response to inorganic substrates, cytokinin metabolism, and disaccharide biosynthesis.

Of 120 genes in P. trichocarpa that responded to I. europaea (PMI82) inoculation, but not M. elongata (PMI93) inoculation, two genes were predicted to be plant LRR (pLRR) (Gene ID: Potri.005G043700 and Potri.019G110800) and were downregulated.

\section{Fungal genes upregulated in response to $P$. trichocarpa.}

Our initial attempts to profile the expression pattern of M. elongata (PMI93) and I. europaea (PMI82) genes in roots and soils were hampered by a limited number of fungal endophyte reads (Fig. 2). For example, only 56,000 paired reads of M. elongata (from more than 28 million qualified reads) were detected from individual soil samples. This may be a general feature of many endophytes, which are characterized by lower activity and abundance especially within plant tissues. Because $M$. elongata expressed higher numbers of mRNA transcripts than did I. europaea for the soil and root samples without inoculum (Fig. 2), the molecular activities of $M$. elongata (PMI93) in the bioassay were investigated further. The higher abundance of $M$. elongata (PMI93) mRNA transcripts in rhizosphere soil (Fig. 2) permitted comparison of relative expression of fungal genes (percent reads of $M$. elongata $=5 \%$, around 1.4 million reads per sand sample). In total, 7,950 genes of $M$. elongata (PMI93) were detected across all (4) biological replicates of sand samples inoculated with $M$. elongata (PMI93). To study the functional categories active in $M$. elongata (PMI93), we investigated the number of transcribed genes and their functional proportions of $M$. elongata (PMI93) detected in sand and in vitro (Supplementary Fig. S9;

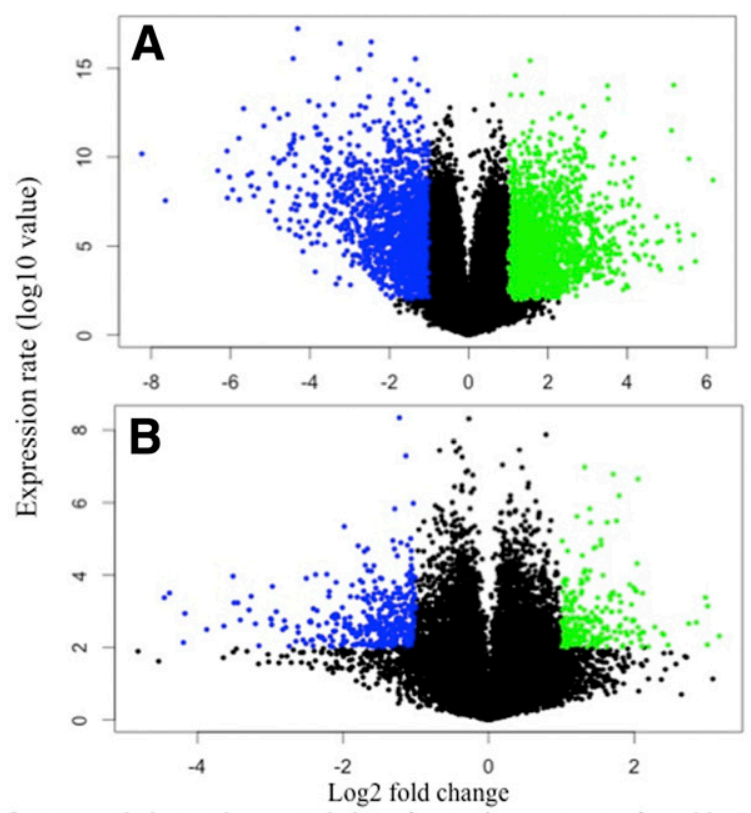

$[+$, up regulation; -, down regulation of genes in response to fungal inoculation)

Fig. 4. Volcano plots elucidate the counts and expression rate of Populus trichocarpa genes up- and downregulated (green and blue dots, respectively) in response to inoculation with A, Mortierella elongata (PMI93) and B, Ilyonectria europaea (PMI82). Black dots represent the expression of genes with no significant difference across the comparisons. Data were generated according to normalized expression rates using Cufflink packages. Data on the loading gene factors were generated using the coordinate scales on the left $(\log 10$ of expression rate) and the bottom (mean of $\log 2$-fold changes). Cross-comparative expression of the genes was analyzed using t-text to compare $P$. trichocarpa with fungal inoculation versus without fungal inoculation ( $n=4 ; P \leq 0.01$; false discovery rate $\leq 0.05$; fold changes $\geq 2$ ). 
Supplementary Dataset S6). In general, a similar pattern and proportion of M. elongata (PMI93) functional genes was detected across sand and culture conditions. However, fewer $M$. elongata genes encoding secreted proteins (fSSP), leucinerich receptors, and WD40 were detected in P. trichocarpa-sand bioassay treatments inoculated with M. elongata (PMI93), compared with $M$. elongata (PMI93) grown axenically in pure culture $(P \leq 0.05)$. Of $87 \mathrm{fSSP}$ detected in the sand with $P$. trichocarpa grown nearby, only $3 \mathrm{fSSP}$ were not detected in the culture (Supplementary Dataset S7). Comparative metatranscriptomics also showed genes for RNA modification, translation, signal transduction, lipid transport and metabolism, and chitinase were significantly upregulated in M. elongata (PMI93) when $M$. elongata (PMI93) grew with P. trichocarpa nearby compared with pure culture.

\section{DISCUSSION}

Some fungal endophytes are known to be beneficial symbiotic microbes able to promote plant growth and induce plant defense (Grelet et al. 2017; Lee et al. 2011; Varma et al. 1999; Zuccaro et al. 2014). In this study, soil inoculation with the

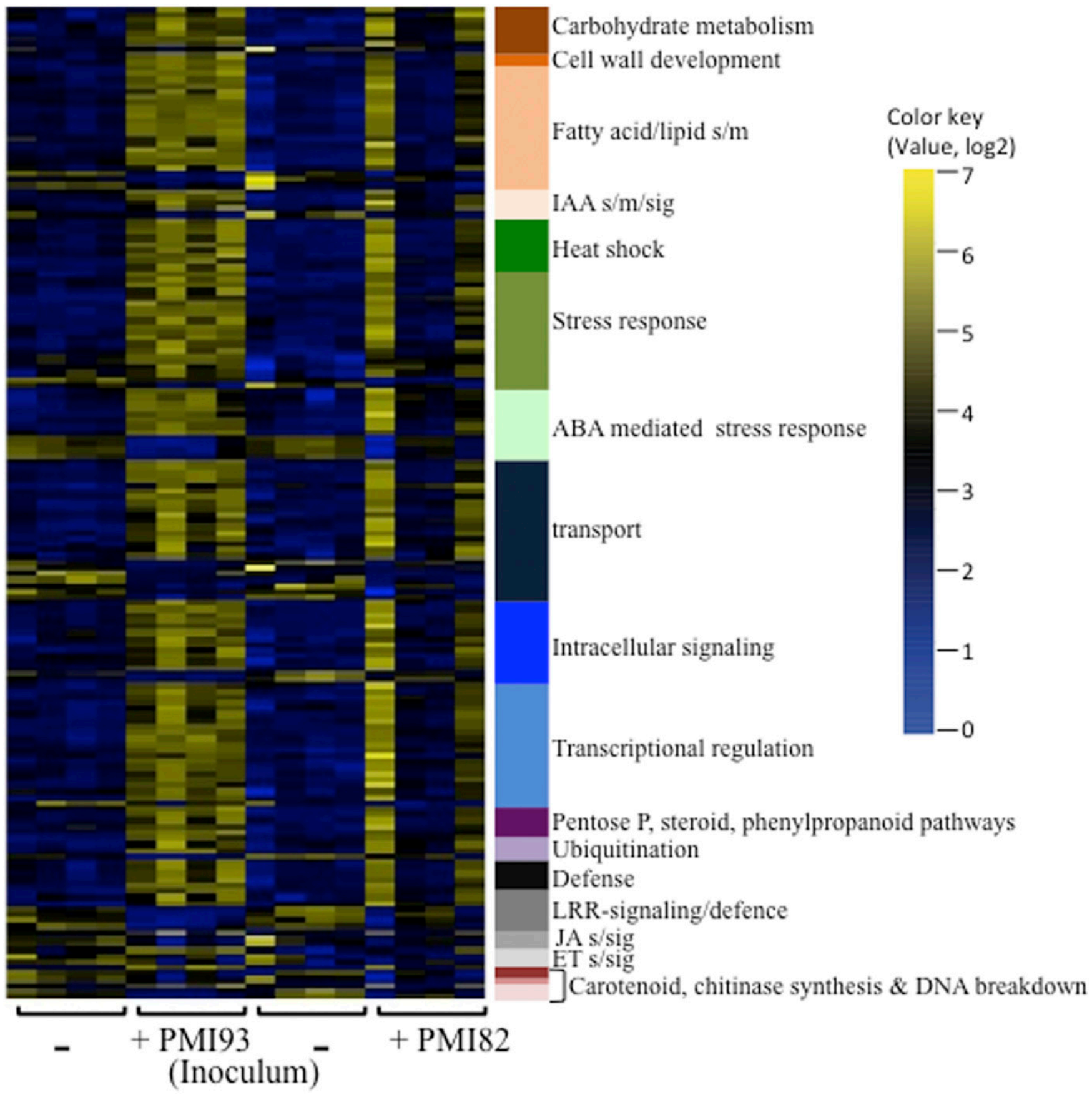

Fig. 5. Populus trichocarpa genes whose expression changes in response to both Mortierella elongata (PMI93) and Ilyonectria europaea (PMI82) inoculation. Significant changes in individual expression between inoculated root $(+)$ versus noninoculated root $(-)$ samples were clustered according to their biological function (greater than twofold changes; false discovery rate $\leq 0.05$; Benjamini-Hochberg test). The color key represents RPKM (reads per kilobase of transcript per million mapped reads) normalized $\log 2$-transformed counts of the genes. The Wilcoxon signed-rank test (Bauer 1972) was applied to filter the data. The genes for other catalytic activities and unknown functions were not included in the heatmap. Each $P$. trichocarpa gene manipulated by the fungal species is given with the expression profiles across the four biological replicates per treatment. 
ubiquitous fungal endophyte M. elongata (PMI93) promoted plant growth, whereas the effects of inoculation with another common endophyte, I. europaea (PMI82), were neutral in phenotype (Fig. 1). RNA-Seq data demonstrated that M. elongata (PMI93) and I. europaea (PMI82) both have a dual life strategies: each can grow as a root endophyte or as a soil saprotroph (Fig. 2). The mycelium of M. elongata (PMI93) forms a biofilm on plant roots, indicating that $M$. elongata (PMI93) can directly interact with plant roots (Supplementary
Fig. S10). The differentiation of an individual fungal mycelial network between two life strategies also implies that a fungal isolate itself may utilize multiple resources while interacting with one or more plant hosts. Fungal endophytes have been categorized into four classes according to their life strategies (Rodriguez et al. 2009). Mortierella spp. and other class 4 fungal endophytes live within the roots of their host plants for at least a part of their life cycle without apparent symptoms (Rodriguez and Redman 1997; Rodriguez et al. 2009; Wilson

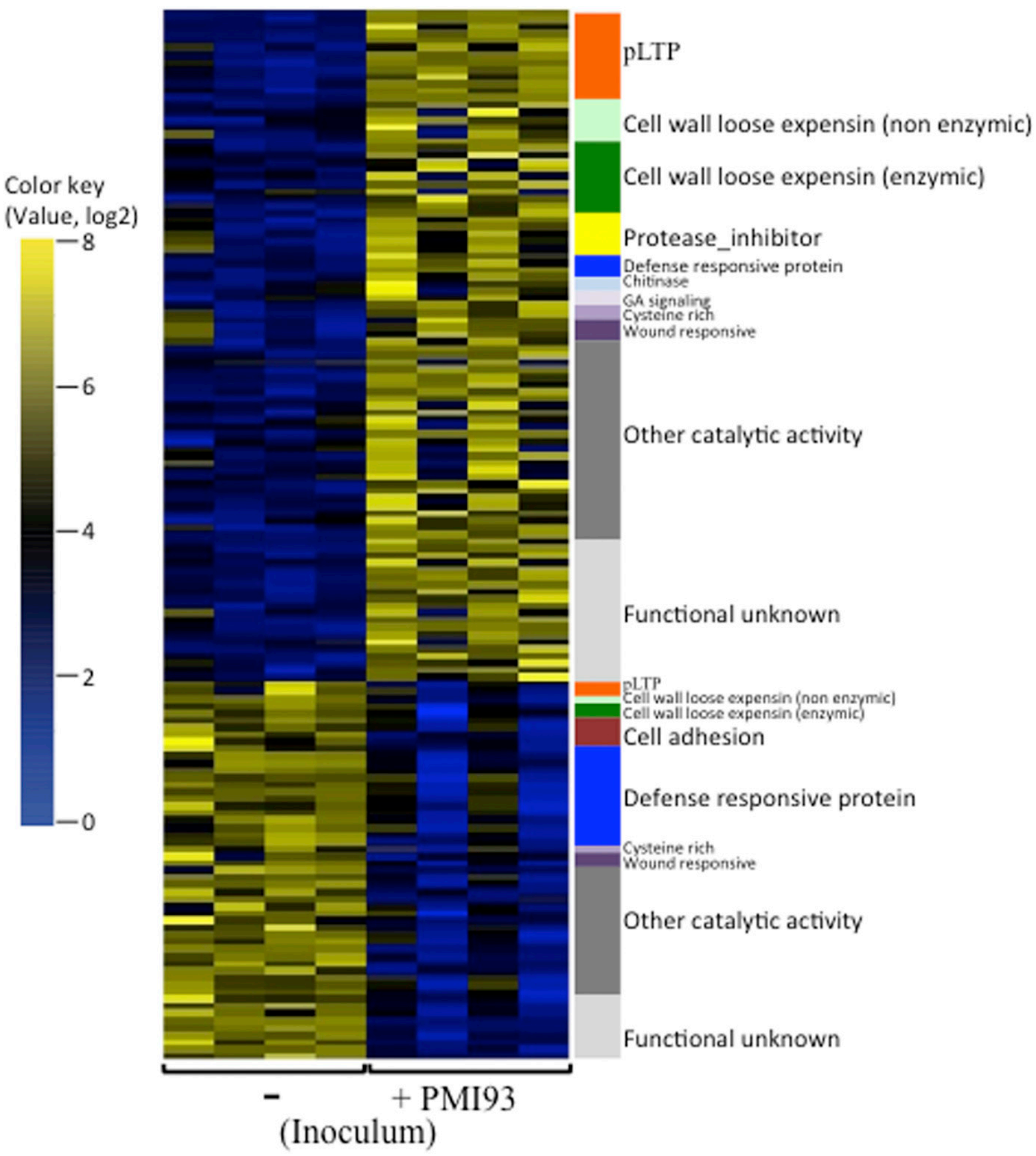

Fig. 6. Significant changes in Populus trichocarpa small secreted protein genes in response to inoculation with Mortierella elongata (PMI93) (greater than twofold changes; false discovery rate $\leq 0.05$ ). The method for data analysis is described in Figure 5. 
1995). In contrast, saprotrophic fungi live off dead organic matter in soils and dead plant tissues. The presence of fungal transcriptomes in roots and soils suggests that $M$. elongata (PMI93) and I. europaea (PMI82) participate in a combination of endophytic and saprotrophic activities (Fig. 2). However, the low proportion of M. elongata and I. europaea transcripts in the soil and root RNA probably reflects the low biomass of these fungi in these two niches. This fact prevented the performance of a detailed analysis of the $M$. elongata transcriptome in response to a host plant.

Results from this study also demonstrated how enrichment of a single fungal taxon can shift the whole community of rootand soil-associated microbes, thus altering the ecological functions of associated plants and diverse soil taxa (Fig. 3). M. elongata (PMI93) may promote plant growth indirectly by manipulating the community and functioning of other rhizosphere microbes (Fig. 3), altering the nutrient composition of soil to facilitate resource acquisition (e.g., nitrogen, lipids), or modulating plant phytohormones (e.g., IAA, GA). The common surveillance genes in $P$. trichocarpa were activated in response to inoculation with both fungal species (Fig. 5), even though the two fungal species played different roles in association with $P$. trichocarpa (Fig. 1). This indicates that $P$. trichocarpa may react to different biotic conditions through a common set of signaling pathways. Since soils collected destructively over a time interval were used to study the effects of M. elongata (PMI93) and I. europaea (PMI82), the possibility that the different responses of $P$. trichocarpa to these two endophytes may have been influenced by interactions between the inoculated endophytes and soil microorganisms cannot be excluded.

Unlike pathogenic or mycorrhizal fungi that utilize a battery of fungal effectors to modulate plant defenses, the $M$. elongata genome has a reduced amount of fSSP (417 fSSP genes in the Morel1 genome) (Supplementary Dataset S8) compared with fungal specialists or obligate biotrophs (around 700 to 1,400 fSSP) (Kim et al. 2016). A similar pattern and proportion of M. elongata (PMI93) fSSP were present in soil compared with plants grown in pure culture. Thus, direct interactions between fungal effectors and plant receptors likely played a lesser role in $P$. trichocarpa-M. elongata interactions.

Populus spp. formed functional symbioses with both AMF and EMF using effector-receptor communication (Martin et al. 2016; Plett et al. 2011). During ectomycorrhizal interaction between $P$. trichocarpa and Laccaria bicolor, a fungal effector fSSP (MiSSP7) was taken up by $P$. trichocarpa and imported into plant nuclei, where it suppressed the JA-mediated plant defense response (Plett et al. 2011, 2014). In the current study, inoculation of $P$. trichocarpa with M. elongata (PMI93) altered plant JA signaling, possibly affecting the suppression of the JAderived pathway. Thus, many beneficial microorganisms, such as M. elongata and Ilyonectria, may use different strategies to interact with their $P$. trichocarpa host.

\section{Enrichment of genes for plant lipid pathway in response to $M$. elongata (PMI93).}

Given that root endophytes do not generally grow within plant cells, interactions between $P$. trichocarpa and its endophytes must occur within extracellular spaces. The proportion of genes encoding predicted pSSP in P. trichocarpa in response to M. elongata (PMI93) is high (approximately 40\%; 147 genes) compared with other genes for extracellular activities. RNA-Seq data indicates that the pLTP are the primary pSSP produced by $P$. trichocarpa in response to M. elongata (PMI93) inoculation (Fig. 6). Only 15 genes for pSSP were differentially expressed in P. trichocarpa in response to I. europaea (PMI82). In addition, genes for fatty acid and lipid biosynthetic processes represent the primary set of $P$. trichocarpa genes enriched in response to $M$. elongata (PMI93), suggesting the involvement of lipids in P. trichocarpa-M. elongata interactions. As mentioned earlier, $P$. trichocarpa genes for pLTP were enriched in response to $M$. elongata (PMI93). These pLTP contain signal peptides that direct their secretion into the extracellular matrix. In addition, the affinity of pLTP for lipids is presumably fundamental to their function. In other plants, pLTP have been shown to be responsible for translocating phospholipids and other fatty acid groups across cell membranes (Kader 1996). They may also bind to ligands that contain acyl groups. Ligand prediction analysis indicated these $P$. trichocarpa $\mathrm{pLTP}$ can bind to saturated fatty acids (e.g., stearic and palmitic acids). The biological roles of pLTP are still unclear; however, several studies suggest their involvement in antimicrobial activity, defensive signaling, cuticle deposition , and cell wall loosening (Yeats and Rose 2008). Growing evidence also suggests that another function of family $1 \mathrm{pLTP}$ is to promote plant cell wall extension (Nieuwland et al. 2005). Upregulation of gene groups for pLTP, 6 expansin-like proteins, and 10 enzymes associated with cell wall loosening in $P$. trichocarpa (Fig. 6) implicates pLTP as potential modulators of nonenzymatic and enzymatic cell wall loosening (Marowa et al. 2016). Similar to other family 1 pLTP (Pagnussat et al. 2012), P. trichocarpa pLTP may function as the extracellular lipid transfer protein and may be relocalized intracellularly to facilitate fatty acid- and lipidassociated pathways.

In a separate study, M. elongata (PMI93) produced polyunsaturated fatty acids under normal growth conditions (Uehling et al. 2017) (Supplementary Fig. S11). The higher number of genes and higher expression rates of lipid transport and metabolism genes in M. elongata (PMI93) detected in sand-grown $P$. trichocarpa cuttings compared with cultured isolates suggests higher induction of M. elongata (PMI93) lipid metabolic activities in the plant-soil system. We hypothesize that lipids produced by $M$. elongata may serve as ligands for pLTP.

\section{M. elongata (PMI93) manipulates P. trichocarpa SSP (pSSP) and LRR (pLRR) genes involved in defense responses.}

In P. trichocarpa, pSSP for defense responses (Fig. 6) and pLRR-RK, were predominantly downregulated in response to M. elongata (PMI93). Three groups of these pSSP were identified: 1) dirigent-like protein; 2) germin-like protein; and 3) PR-thaumatin-associated protein. Ligand prediction analysis showed that these pSSP are able to bind diverse ligands, implying that different parts of the plant immune system were suppressed, including JA, SA, hypersensitive response (HR), and LRR-RK-associated defense response (explained below).

Enzyme active-site prediction showed that dirigent-like pSSP contained the activity of allene oxide cyclase (AOC). These dirigent-like pSSP contained an eight-stranded antiparallel $\beta$-barrel with a central hydrophobic ligand-binding site. The predicted ligands of dirigent-like pSSP included reaction intermediates required for allene oxide synthase (AOS) activities (i.e., enoic and vernolic acids) (Wasternack and Kombrink 2010). The essential function of AOC and AOS in JA biosynthesis has been reported in other studies (Ishiga et al. 2003; Park et al. 2002; von Malek et al. 2002; Wasternack 2007), implicating manipulation by $M$. elongata (PMI93) in the suppression of JA-mediated plant defense. Enzyme active-site prediction suggests that germin-like pSSP contained oxalate oxidase $(\mathrm{OXO})$ activity, which can catalyze the conversion between oxalate and $\mathrm{CO}_{2}+\mathrm{H}_{2} \mathrm{O}_{2}$. The $\mathrm{OXO}$ are involved in hypersensitive plant cell death (Lane 2002) and increase their activity under biotic stress (Hurkman and Tanaka 1996; Zhou et al. 1998). Together these results suggest the possibility that 
M. elongata (PMI93) can downregulate the HR of its $P$. trichocarpa host. Downregulation of several PR-thaumatin-associated proteins with $\beta$-1,3-glucanases activity showed that M. elongata (PMI93) may suppress multiple routes of plant immunity, including the SA-mediated pathway (Liu et al. 2010) and fungal cell wall degradation (Kuć 1995; Lusso and Kuć 1996).

Plant membrane-localized receptor kinases play important roles in sensing and responding to environmental signals (Osakabe et al. 2013). These receptors perceive the extracellular ligands to phosphorylate intracellular kinase domains to activate downstream pathways. Within the $P$. trichocarpa genome, pLRR-RK account for the largest group of membranelocalized receptor kinases. pLRR-RK can exhibit diverse biological functions, and most have been shown to play a role in plant defense (McHale et al. 2006). Downregulation of P. trichocarpa $\mathrm{pLRR}-\mathrm{RK}$ raises the possibility that $M$. elongata (PMI93) can balance pLRR-RK signaling and growth tradeoffs to optimize plant fitness.

\section{Conclusions.}

In conclusion, our findings show differential responses of P. trichocarpa to two functionally different fungal endophytes, M. elongata (PMI93) and I. europaea (PMI82). These two fungal endophytes have endophytic and saprotrophic activities. The enrichment of a single fungal taxon (PMI93 or PMI82) can shift the whole community of root- and soil-associated microbes and can alter the gene expression of their host plant. RNA-Seq results suggest that $M$. elongata (PMI93) can modulate $P$. trichocarpa defense responses, nutrient uptake, and photosynthetic-associated energy production through direct or indirect interactions with its host. In particular, the presence of M. elongata (PMI93) leads to downregulation of genes involved in plant immune response and hormone signaling (e.g., JA, SA, and ET signaling, HR, and fungal cell wall degradation) and alters expression of genes involved in GA- and lipidassociated pathways, which may result in the observed growth enhancement. Future studies may consider targeting lipidderived communication and metabolism between $M$. elongata and $P$. trichocarpa roots to better understand the interactions of these symbionts.

\section{MATERIALS AND METHODS}

\section{Inoculum preparation.}

Sterile millet seeds were used as the medium to sustain viability and growth of fungal isolates. To prepare sterile millet seeds for fungal inoculum, millet seeds were soaked overnight in sterile distilled water. The excess water was drained, and the wet millet seeds were transferred to mushroom spawn bags with micropore patch. All bags were tightly closed and autoclaved at $120^{\circ} \mathrm{C}$ and $15 \mathrm{psi}$ for $45 \mathrm{~min}$ for two cycles and cooled between cycles.

Prior to inoculating spawn bags, fungal mycelium (M. elongata [PMI93] and I. europaea [PMI82]) was grown on pure modified Melin Norkrans (MMN) $1 \%$ agar media at $25^{\circ} \mathrm{C}$ (Rossi and Oliveira 2011). After 3 days of culturing, the fungal biomass with solid media was finely chopped into small cubes (around $3 \times 3 \times 3 \mathrm{~mm}^{3}$ ) and transferred to sterile culture bags containing sterile millet seeds. After 1 month of incubation at $25^{\circ} \mathrm{C}$, the fungi had completely colonized the millet seeds used as inoculum in soil bioassay experiments.

\section{Soil bioassay and sample collection for metatranscriptomics.}

Fresh cuttings of $P$. trichocarpa were collected from Washington and Oregon in spring 2015, including four genotypes:
BESC86, GW7974, BESC4, and BESC320. The 30-cm long vegetative cuttings of $P$. trichocarpa were submerged in tap water for 3 days, with daily water changes. Prior to further use cuttings were surface-sterilized by soaking in 5 to $7 \%$ bleach solution with $0.01 \%$ Tween 20 for 15 to $20 \mathrm{~min}$ and then rinsed with sterile distilled water. Cuttings were planted in sterile sand and allowed to root under fluorescent lighting and regular watering. After 1 month of growth, the plants were transferred on the same day to 1) soil collected from forest sites and diluted with sterile sand: soil/sand at 30:70 (wt/wt) ratio; and 2) sterile sand only to serve as a "no soil control (sand bioassay)." Because fungal inocula grew at different rates, two different fresh soil samples from the same field site were used for inoculation with M. elongata (PMI93) and I. europaea (PMI82). Potted plants were inoculated in replicate with $M$. elongata (PMI93) and I. europaea (PMI82) (soil/millet inoculum at 99:1 [wt/wt] ratio). Negative control treatments used sterile millet mixed with each soil. Inoculated plants were placed in a growth chamber at $25^{\circ} \mathrm{C}$, $80 \%$ humidity, and fluorescent light at $200 \mu \mathrm{mol} \mathrm{m} \mathrm{m}^{-2} \mathrm{~s}^{-1}$ for 16 h/day. After 2 to 3 months, some replicates of $P$. trichocarpa growing in sand only without M. elongata (PMI93) inoculum did not survive and could not be used for further studies (Fig. 1). After 4 months of growth, roots and soils (four biological replicates each) collected from the P. trichocarpa BESC4 soil bioassay and sand bioassay (with PMI93 inoculum) were harvested for metatranscriptomic analysis. Physiological measurements for each plant are presented in Figure 1.

\section{RNA preparation, cDNA library construction, and sequencing.}

Total RNA from roots and soils was extracted following a cetyl trimethylammonium bromide and chloroform extraction and $\mathrm{LiCl}$ precipitation protocol described previously (Liao et al. 2014). The mRNA and cDNA for RNA-Seq analysis were purified using a TruSeq RNA sample preparation kit (Illumina, San Diego, CA). cDNA pools were sequenced on Illumina HiSeq 2000 instruments in the Duke Center for Genomic and Computational Biology. Twelve samples were sequenced in an individual lane to generate a total of approximately $40 \mathrm{~Gb}$ of data. In total, 32 samples (roots and soils) in soil bioassay, four samples in sand bioassay, and three fungal culture samples were sequenced for this study. RNA-Seq data have been deposited at the NCBI Short Read Archive (SRP057033).

\section{Sequence assembly and annotation.}

Genome sequences produced by the Joint Genome Institute for $P$. trichocarpa v3.1 (Tuskan et al. 2006), M. elongata AG77 v1 (Morel1) (Uehling et al. 2017), and I. europaea v1.0 were used as references for RNA-Seq filtered read mapping using TopHat and Cufflink packages (Trapnell et al. 2009, 2010). The genome and transcriptome of I. europaea were sequenced using an Illumina platform. The pipelines applied for the assembly of the I. europaea genome are described in the Supplementary Text. A rRNA mapping method was employed to sort reads for all other fungal rRNA as well (Liao et al. 2014, 2016). Recovered rRNA reads containing D1/D2 regions were used to calculate the relative abundance of fungal communities. Recent studies indicate that rRNA reads recovered from metatranscriptome (poly-A enrichment strategy) and RNA-based amplicon sequencing detected similar trends of microbial diversity and community (Chen et al. 2018; Liao et al. 2014). Nonmetric multidimensional scaling (NMDS) was performed on both Euclidean and Bray-Curtis dissimilarity matrices, and results from multiple different dimensions were examined. Differences in community composition among the treatments (with and without fungal inoculation) were tested using permutational multivariate analysis of variance (PERMANOVA). 
Results for PERMANOVA were corrected for multiple comparison using FDR. $P$ values were calculated based on pseudo- $F$ statistics, and results with $P \leq 0.05$ were considered statistically significant. Both NMDS and PERMANOVA were performed using Vegan package version 2.5.3 in R (3.5.1). They are performed using metaMDS and adonis functions, respectively. Comparative metatranscriptomics using Cuffdiff and Cuffcompare packages were applied to identify key plant genes that differentially respond to fungal inoculation (Trapnell et al. 2010). An FDR of 5\% was used to identify highly expressed transcripts with at least twofold change for the genes. A combination of GO (Ashburner et al. 2000), KEGG (Kanehisa et al. 2012), and KOG (Tatusov et al. 2003) packages was used for gene annotation for P. trichocarpa v3.1. Gene-enrichment analysis was applied using the ClueGO platform (Bindea et al. 2009, 2013). It is important to note that only around $18 \%$ of the P. trichocarpa genes in the $P$. trichocarpa genomes were assigned to the KEGG and GO categories. For example, of 60,000 P. trichocarpa genes, 10,876 genes were assigned to 7,068 GO numbers. In addition to geneenrichment analysis, several other software packages were used to better annotate domains to identify the subcellular locations of genes with an unknown function or predicted extracellular enzymes. For domain analysis, EMBL-EBI, Phobius (Käll et al. 2004), Signal-3L (Shen and Chou 2007; Zhang and Shen 2017), Signal P v4.1 (Kihara 2017), and TMHMM v2.0 (Krogh et al. 2001) were used for the prediction of signal peptides and transmembrane helix domains. Endoplasmic reticulum (ER) retention signal ScanPrositeTool (de Castro et al. 2006) and Euk-mPLoc 2.0 (Chou and Shen 2010) were used to identify the subcellular localization of contigs. SSP were defined as having 1) a size smaller than 300 amino acids (detected manually); 2) signal peptide predicted at the $\mathrm{N}$ end (Signal-P v4.1); 3) extracellular location (Euk-mPLoc 2.0); 4) no transmembrane domains (EukmPLoc 2.0, TMHMM v2.0, EMBL-EBI, and Signal-3L); and 5) no ER retention motifs (ER retention signal_ScanPrositeTool). The tertiary structures, ligand-binding sites, and enzyme activation sites of the individual SSP were predicted using ITASSER v3.0 (Roy et al. 2010; Yang and Zhang 2015; Yang et al. 2015; Zhang 2008). With this approach, we were able to assign the majority of contigs with unknown function to either SSP or transmembrane proteins. Plots (Fig. 4) and heatmaps (Figs. 5 and 6) were generated with statistical packages in $\mathrm{R}(\mathrm{R}$ Development Core Team 2003). Given that different batches of soil were used for M. elongata (PMI93) and I. europaea (PMI82) inoculations, we did not attempt to compare the differential responses of plants between the two fungal species. Only the expression of $P$. trichocarpa genes that were manipulated by both fungal species are presented (Fig. 5). The greater number of plant genes responsive to M. elongata (PMI93) is reported in Figure 6.

\section{ACKNOWLEDGMENTS}

We thank C.-M. (Jimmy) Hsu for help monitoring and measuring Populus trichocarpa plants, Lee Gunter for providing $P$. trichocarpa cuttings, and members of the Plant-Microbe Interfaces Science Focus Area for input during all phases of this study.

\section{LITERATURE CITED}

Ashburner, M., Ball, C. A., Blake, J. A., Botstein, D., Butler, H., Cherry, J. M., Davis, A. P., Dolinski, K., Dwight, S. S., Eppig, J. T., Harris, M. A., Hill, D. P., Issel-Tarver, L., Kasarskis, A., Lewis, S., Matese, J. C., Richardson, J. E., Ringwald, M., Rubin, G. M., and Sherlock, G.; The Gene Ontology Consortium. 2000. Gene ontology: Tool for the unification of biology. Nat. Genet. 25:25-29.

Bauer, D. F. 1972. Constructing confidence sets using rank statistics. J. Am. Stat. Assoc. 67:687-690

Baum, C., and Makeschin, F. 2000. Effects of nitrogen and phosphorus fertilization on mycorrhizal formation of two poplar clones (Populus trichocarpa and $P$. tremula $\times$ tremuloides). J. Plant Nutr. Soil Sci. 163: 491-497.

Bidartondo, M. I., Read, D. J., Trappe, J. M., Merckx, V., Ligrone, R., and Duckett, J. G. 2011. The dawn of symbiosis between plants and fungi. Biol. Lett. 7:574-577.

Bindea, G., Galon, J., and Mlecnik, B. 2013. CluePedia Cytoscape plugin: Pathway insights using integrated experimental and in silico data. Bioinformatics 29:661-663.

Bindea, G., Mlecnik, B., Hackl, H., Charoentong, P., Tosolini, M., Kirilovsky, A., Fridman, W.-H., Pagès, F., Trajanoski, Z., and Galon, J. 2009. ClueGO: A Cytoscape plug-in to decipher functionally grouped gene ontology and pathway annotation networks. Bioinformatics 25: 1091-1093.

Bois, G., Piché, Y., Fung, M. Y. P., and Khasa, D. P. 2005. Mycorrhizal inoculum potentials of pure reclamation materials and revegetated tailing sands from the Canadian oil sand industry. Mycorrhiza 15:149-158.

Bonito, G., Hameed, K., Ventura, R., Krishnan, J., Schadt, C. W., and Vilgalys, R. 2016. Isolating a functionally relevant guild of fungi from the root microbiome of Populus. Fungal Ecol. 22:35-42.

Bonito, G., Reynolds, H., Robeson, M. S., 2nd, Nelson, J., Hodkinson, B. P. Tuskan, G., Schadt, C. W., and Vilgalys, R. 2014. Plant host and soil origin influence fungal and bacterial assemblages in the roots of woody plants. Mol. Ecol. 23:3356-3370.

Cabral, A., Rego, C., Nascimento, T., Oliveira, H., Groenewald, J. Z., and Crous, P. W. 2012. Multi-gene analysis and morphology reveal novel Ilyonectria species associated with black foot disease of grapevines. Fungal Biol. 116:62-80.

Chaverri, P., Salgado, C., Hirooka, Y., Rossman, A. Y., and Samuels, G. J. 2011. Delimitation of Neonectria and Cylindrocarpon (Nectriaceae, Hypocreales, Ascomycota) and related genera with Cylindrocarponlike anamorphs. Stud. Mycol. 68:57-78.

Chou, K.-C., and Shen, H.-B. 2010. A new method for predicting the subcellular localization of eukaryotic proteins with both single and multiple sites: Euk-mPLoc 2.0. PLoS One 5:e9931.

Chen, K.-H., Liao, H.-L., Arnold, A. E., Bonito, G., and Lutzoni, F. 2018 RNA-based analyses reveal fungal communities structured by a senescence gradient in the moss Dicranum scoparium and the presence of putative multi-trophic fungi. New Phytol. 218:1597-1611.

Cregger, M. A., Veach, A. M., Yang, Z. K., Crouch, M. J., Vilgalys, R., Tuskan, G. A., and Schadt, C. W. 2018. The Populus holobiont: Dissecting the effects of plant niches and genotype on the microbiome. Microbiome 6:31.

Cui, J.-L., Guo, T.-T., Ren, Z.-X., Zhang, N.-S., and Wang, M.-L. 2015. Diversity and antioxidant activity of culturable endophytic fungi from alpine plants of Rhodiola crenulata, $R$. angusta, and $R$. sachalinensis. PLoS One 10:e0118204.

de Castro, E., Sigrist, C. J. A., Gattiker, A., Bulliard, V., LangendijkGenevaux, P. S., Gasteiger, E., Bairoch, A., and Hulo, N. 2006 ScanProsite: Detection of PROSITE signature matches and ProRuleassociated functional and structural residues in proteins. Nucleic Acids Res. 34(Web Server Issue):W362-W365.

Farh, M. E.-A., Kim, Y.-J., Kim, Y.-J., and Yang, D.-C. 2018 Cylindrocarpon destructans/Ilyonectria radicicola-species complex: Causative agent of ginseng root-rot disease and rusty symptoms. J. Ginseng Res. 42:9-15.

Gottel, N. R., Castro, H. F., Kerley, M., Yang, Z., Pelletier, D. A., Podar, M., Karpinets, T., Uberbacher, E., Tuskan, G. A., Vilgalys, R., Doktycz, M. J., and Schadt, C. W. 2011. Distinct microbial communities within the endosphere and rhizosphere of Populus deltoides roots across contrasting soil types. Appl. Environ. Microbiol. 77:5934-5944.

Grelet, G.-A., Ba, R., Goeke, D. F., Houliston, G. J., Taylor, A. F. S., and Durall, D. M. 2017. A plant growth-promoting symbiosis between Mycena galopus and Vaccinium corymbosum seedlings. Mycorrhiza 27:831-839.

Guevara, G., Bonito, G., Trappe, J. M., Cázares, E., Williams, G., Healy, R. A., Schadt, C., and Vilgalys, R. 2013. New North American truffles (Tuber spp.) and their ectomycorrhizal associations. Mycologia 105: 194-209.

Hersh, M. H., Vilgalys, R., and Clark, J. S. 2012. Evaluating the impacts of multiple generalist fungal pathogens on temperate tree seedling survival. Ecology 93:511-520.

Hurkman, W. J., and Tanaka, C. K. 1996. Germin gene expression is induced in wheat leaves by powdery mildew infection. Plant Physiol. 111:735-739.

Ishiga, Y., Inagaki, Y., Toyoda, K., Shiraishi, T., and Ichinose, Y. 2003. Expression of allene oxide synthase and allene oxide cyclase in the interactions between pea and fungal pathogens. J. Gen. Plant Pathol. 69: 351-357. 
Johnson, J. M., Ludwig, A., Furch, A., Mithöfer, A., Scholz, S., Reichelt, M., and Oelmüller, R. 2019. The beneficial root-colonizing fungus Mortierella hyalina promotes the aerial growth of Arabidopsis and activates calciumdependent responses which restrict Alternaria brassicae-induced disease development in roots. Mol. Plant-Microbe Interact. 32:351-363.

Kader, J.-C. 1996. Lipid-transfer proteins in plants. Annu. Rev. Plant Physiol. Plant Mol. Biol. 47:627-654.

Käll, L., Krogh, A., and Sonnhammer, E. L. L. 2004. A combined transmembrane topology and signal peptide prediction method. J. Mol. Biol. 338:1027-1036.

Kanehisa, M., Goto, S., Sato, Y., Furumichi, M., and Tanabe, M. 2012. KEGG for integration and interpretation of large-scale molecular data sets. Nucleic Acids Res. 40(Database Issue):D109-D114.

Kihara, D., ed. 2017. Protein Function Prediction: Methods and Protocols. Springer, New York.

Kim, K.-T., Jeon, J., Choi, J., Cheong, K., Song, H., Choi, G., Kang, S., and Lee, Y.-H. 2016. Kingdom-wide analysis of fungal small secreted proteins (SSPs) reveals their potential role in host association. Front. Plant Sci. 7:186.

Krogh, A., Larsson, B., von Heijne, G., and Sonnhammer, E. L. 2001. Predicting transmembrane protein topology with a hidden Markov model: Application to complete genomes. J. Mol. Biol. 305:567-580.

Kuć, J. 1995. Phytoalexins, stress metabolism, and disease resistance in plants. Annu. Rev. Phytopathol. 33:275-297.

Kwaśna, H., Szewczyk, W., and Behnke-Borowczyk, J. 2016. Fungal root endophytes of Quercus robur subjected to flooding. For. Pathol. 46:35-46

Lane, B. G. 2002. Oxalate, germins, and higher-plant pathogens. IUBMB Life 53:67-75.

Lee, Y.-C., Johnson, J. M., Chien, C.-T., Sun, C., Cai, D., Lou, B., Oelmüller, R., and Yeh, K.-W. 2011. Growth promotion of Chinese cabbage and Arabidopsis by Piriformospora indica is not stimulated by mycelium-synthesized auxin. Mol. Plant-Microbe Interact 24:421-431.

Liao, H.-L., Chen, Y., Bruns, T. D., Peay, K. G., Taylor, J. W., Branco, S., Talbot, J. M., and Vilgalys, R. 2014. Metatranscriptomic analysis of ectomycorrhizal roots reveals genes associated with Piloderma-Pinus symbiosis: Improved methodologies for assessing gene expression in situ. Environ. Microbiol. 16:3730-3742.

Liao, H.-L., Chen, Y., and Vilgalys, R. 2016. Metatranscriptomic study of common and host-specific patterns of gene expression between pines and their symbiotic ectomycorrhizal fungi in the genus Suillus. PLoS Genet. 12:e1006348.

Liu, B., Xue, X., Cui, S., Zhang, X., Han, Q., Zhu, L., Liang, X., Wang, X., Huang, L., Chen, X., and Kang, Z. 2010. Cloning and characterization of a wheat $\beta$-1,3-glucanase gene induced by the stripe rust pathogen Puccinia striiformis f. sp. tritici. Mol. Biol. Rep. 37:1045-1052.

Lodge, D. J. 1989. The influence of soil moisture and flooding on formation of VA-endo- and ectomycorrhizae in Populus and Salix. Plant Soil 117: 243-253.

Lusso, M., and Kuć, J. 1996. The effect of sense and antisense expression of the PR-N gene for $\beta$-1,3-glucanase on disease resistance of tobacco to fungi and viruses. Physiol. Mol. Plant Pathol. 49:267-283.

Marowa, P., Ding, A., and Kong, Y. 2016. Expansins: Roles in plant growth and potential applications in crop improvement. Plant Cell Rep. 35: 949-965.

Martin, F., Kohler, A., Murat, C., Veneault-Fourrey, C., and Hibbett, D. S. 2016. Unearthing the roots of ectomycorrhizal symbioses. Nat. Rev. Microbiol. 14:760-773.

McHale, L., Tan, X., Koehl, P., and Michelmore, R. W. 2006. Plant NBSLRR proteins: Adaptable guards. Genome Biol. 7:212.

Nguyen, N. H., Song, Z., Bates, S. T., Branco, S., Tedersoo, L., Menke, J., Schilling, J. S., and Kennedy, P. G. 2016. FUNGuild: An open annotation tool for parsing fungal community datasets by ecological guild. Fungal Ecol. 20:241-248.

Nieuwland, J., Feron, R., Huisman, B. A. H., Fasolino, A., Hilbers, C. W., Derksen, J., and Mariani, C. 2005. Lipid transfer proteins enhance cell wall extension in tobacco. Plant Cell 17:2009-2019.

Osakabe, Y., Osakabe, K., and Shinozaki, K. 2013. Plant environmental stress responses for survival and biomass enhancement. Pages 79-108 in: Climate Change and Plant Abiotic Stress Tolerance. N. Tuteja and S. S. Gill, eds. Wiley-VCH Verlag GmbH \& Co. KGaA, Weinheim, Germany.

Pagnussat, L., Burbach, C., Baluska, F., and de la Canal, L. 2012. An extracellular lipid transfer protein is relocalized intracellularly during seed germination. J. Exp. Bot. 63:6555-6563.

Park, J.-H., Halitschke, R., Kim, H. B., Baldwin, I. T., Feldmann, K. A., and Feyereisen, R. 2002. A knock-out mutation in allene oxide synthase results in male sterility and defective wound signal transduction in Arabidopsis due to a block in jasmonic acid biosynthesis. Plant J. 31:1-12.

Plett, J. M., Daguerre, Y., Wittulsky, S., Vayssières, A., Deveau, A., Melton, S. J., Kohler, A., Morrell-Falvey, J. L., Brun, A., Veneault-Fourrey, C., and Martin, F. 2014. Effector MiSSP7 of the mutualistic fungus Laccaria bicolor stabilizes the Populus JAZ6 protein and represses jasmonic acid (JA) responsive genes. Proc. Natl. Acad. Sci. U.S.A. 111:8299-8304.

Plett, J. M., Kemppainen, M., Kale, S. D., Kohler, A., Legué, V., Brun, A., Tyler, B. M., Pardo, A. G., and Martin, F. 2011. A secreted effector protein of Laccaria bicolor is required for symbiosis development. Curr. Biol. 21:1197-1203.

Podila, G. K., Sreedasyam, A., and Muratet, M. A. 2009. Populus rhizosphere and the ectomycorrhizal interactome. Crit. Rev. Plant Sci. 28:359-367.

R Development Core Team. 2003. The R Reference Manual: Base Package. Network Theory Ltd.,Godalming, U.K.

Rodriguez, R. J., and Redman, R. S. 1997. Fungal life-styles and ecosystem dynamics: Biological aspects of plant pathogens, plant endophytes and saprophytes. Adv. Bot. Res. 24:169-193.

Rodriguez, R. J., White, J. F., Jr., Arnold, A. E., and Redman, R. S. 2009. Fungal endophytes: Diversity and functional roles. New Phytol. 182: 314-330.

Rossi, M. J., and Oliveira, V. L. 2011. Growth of the ectomycorrhizal fungus Pisolithus microcarpus in different nutritional conditions. Braz. J. Microbiol. 42:624-632.

Roy, A., Kucukural, A., and Zhang, Y. 2010. I-TASSER: A unified platform for automated protein structure and function prediction. Nat. Protoc. 5:725-738.

Shakya, M., Gottel, N., Castro, H., Yang, Z. K., Gunter, L., Labbé, J., Muchero, W., Bonito, G., Vilgalys, R., Tuskan, G., Podar, M., and Schadt, C. W. 2013. A multifactor analysis of fungal and bacterial community structure in the root microbiome of mature Populus deltoides trees. PLoS One 8:e76382.

Shen, H.-B., and Chou, K.-C. 2007. Signal-3L: A 3-layer approach for predicting signal peptides. Biochem. Biophys. Res. Commun. 363: 297-303.

Spatafora, J. W., Chang, Y., Benny, G. L., Lazarus, K., Smith, M. E., Berbee, M. L., Bonito, G., Corradi, N., Grigoriev, I., Gryganskyi, A., James, T. Y., O’Donnell, K., Roberson, R. W., Taylor, T. N., Uehling, J., Vilgalys, R., White, M. M., and Stajich, J. E. 2016. A phylum-level phylogenetic classification of zygomycete fungi based on genomescale data. Mycologia 108:1028-1046.

Strullu-Derrien, C., Selosse, M.-A., Kenrick, P., and Martin, F. M. 2018. The origin and evolution of mycorrhizal symbioses: From palaeomycology to phylogenomics. New Phytol. 220:1012-1030.

Swett, C. L., and Gordon, T. R. 2017. Exposure to a pine pathogen enhances growth and disease resistance in Pinus radiata seedlings. For. Pathol. 47:e12298.

Tatusov, R. L., Fedorova, N. D., Jackson, J. D., Jacobs, A. R., Kiryutin, B., Koonin, E. V., Krylov, D. M., Mazumder, R., Mekhedov, S. L., Nikolskaya, A. N., Rao, B. S., Smirnov, S., Sverdlov, A. V., Vasudevan, S., Wolf, Y. I., Yin, J. J., and Natale, D. A. 2003. The COG database: An updated version includes eukaryotes. BMC Bioinformatics 4:41.

Trapnell, C., Pachter, L., and Salzberg, S. L. 2009. TopHat: Discovering splice junctions with RNA-Seq. Bioinformatics 25:1105-1111.

Trapnell, C., Williams, B. A., Pertea, G., Mortazavi, A., Kwan, G., van Baren, M. J., Salzberg, S. L., Wold, B. J., and Pachter, L. 2010. Transcript assembly and quantification by RNA-Seq reveals unannotated transcripts and isoform switching during cell differentiation. Nat. Biotechnol. 28:511-515.

Tuskan, G. A., Difazio, S., Jansson, S., Bohlmann, J., Grigoriev, I., Hellsten, U., Putnam, N., Ralph, S., Rombauts, S., Salamov, A., Schein, J., Sterck, L., Aerts, A., Bhalerao, R. R., Bhalerao, R. P., Blaudez, D., Boerjan, W., Brun, A., Brunner, A., Busov, V., Campbell, M., Carlson, J., Chalot, M., Chapman, J., Chen, G.-L., Cooper, D., Coutinho, P. M., Couturier, J., Covert, S., Cronk, Q., Cunningham, R., Davis, J., Degroeve, S., Déjardin, A., Depamphilis, C., Detter, J., Dirks, B., Dubchak, I., Duplessis, S., Ehlting, J., Ellis, B., Gendler, K., Goodstein, D., Gribskov, M., Grimwood, J., Groover, A., Gunter, L., Hamberger, B., Heinze, B., Helariutta, Y., Henrissat, B., Holligan, D., Holt, R., Huang, W., Islam-Faridi, N., Jones, S., Jones-Rhoades, M., Jorgensen, R., Joshi, C., Kangasjärvi, J., Karlsson, J., Kelleher, C., Kirkpatrick, R., Kirst, M., Kohler, A., Kalluri, U., Larimer, F., Leebens-Mack, J., Leplé, J.-C., Locascio, P., Lou, Y., Lucas, S., Martin, F., Montanini, B., Napoli, C., Nelson, D. R., Nelson, C., Nieminen, K., Nilsson, O., Pereda, V., Peter, G., Philippe, R., Pilate, G., Poliakov, A., Razumovskaya, J., Richardson, P., Rinaldi, C., Ritland, K., Rouzé, P., Ryaboy, D., Schmutz, J., Schrader, J., Segerman, B., Shin, H., Siddiqui, A., Sterky, F., Terry, A., Tsai, C.-J., Uberbacher, E., Unneberg, P., Vahala, J., Wall, K., Wessler, S., Yang, G., Yin, T., Douglas, C., Marra, M., Sandberg, G., Van de Peer, Y., and Rokhsar, D. 2006. The genome of black 
cottonwood, Populus trichocarpa (Torr. \& Gray). Science 313: 1596-1604.

Uehling, J., Gryganskyi, A., Hameed, K., Tschaplinski, T., Misztal, P. K., Wu, S., Desirò, A., Vande Pol, N., Du, Z., Zienkiewicz, A., Zienkiewicz, K., Morin, E., Tisserant, E., Splivallo, R., Hainaut, M., Henrissat, B., Ohm, R., Kuo, A., Yan, J., Lipzen, A., Nolan, M., LaButti, K., Barry, K. Goldstein, A. H., Labbé, J., Schadt, C., Tuskan, G., Grigoriev, I., Martin, F., Vilgalys, R., and Bonito, G. 2017. Comparative genomics of Mortierella elongata and its bacterial endosymbiont Mycoavidus cysteinexigens. Environ. Microbiol. 19:2964-2983.

Vadivelan, G., and Venkateswaran, G. 2014. Production and enhancement of omega-3 fatty acid from Mortierella alpina CFR-GV15: Its food and therapeutic application. BioMed Res. Int. 2014:657414.

Varma, A., Verma, S., Sudha, Sahay, N., Bütehorn, B., and Franken, P. 1999. Piriformospora indica, a cultivable plant-growth-promoting root endophyte. Appl. Environ. Microbiol. 65:2741-2744.

von Malek, B., van der Graaff, E., Schneitz, K., and Keller, B. 2002. The Arabidopsis male-sterile mutant dde2-2 is defective in the ALLENE OXIDE SYNTHASE gene encoding one of the key enzymes of the jasmonic acid biosynthesis pathway. Planta 216:187-192.

Wani, Z. A., Kumar, A., Sultan, P., Bindu, K., Riyaz-Ul-Hassan, S., and Ashraf, N. 2017. Mortierella alpina CS10E4, an oleaginous fungal endophyte of Crocus sativus L. enhances apocarotenoid biosynthesis and stress tolerance in the host plant. Sci. Rep. 7:8598.

Wasternack, C. 2007. Jasmonates: An update on biosynthesis, signal transduction and action in plant stress response, growth and development. Ann. Bot. 100:681-697.

Wasternack, C., and Kombrink, E. 2010. Jasmonates: Structural requirements for lipid-derived signals active in plant stress responses and development. ACS Chem. Biol. 5:63-77.
Wilson, D. 1995. Endophyte: The evolution of a term, and clarification of its use and definition. Oikos 73:274-276.

Wu, Y., Zhang, D., Chu, J. Y., Boyle, P., Wang, Y., Brindle, I. D., De Luca, V., and Després, C. 2012. The Arabidopsis NPR1 protein is a receptor for the plant defense hormone salicylic acid. Cell Rep.1:639-647.

Yang, J., Yan, R., Roy, A., Xu, D., Poisson, J., and Zhang, Y. 2015. The ITASSER Suite: Protein structure and function prediction. Nat. Methods 12:7-8.

Yang, J., and Zhang, Y. 2015. Protein structure and function prediction using I-TASSER. Curr. Protoc. Bioinformatics 52:5.8.1-5.8.15.

Yang, X., Tschaplinski, T. J., Hurst, G. B., Jawdy, S., Abraham, P. E., Lankford, P. K., Adams, R. M., Shah, M. B., Hettich, R. L., Lindquist, E., Kalluri, U. C., Gunter, L. E., Pennacchio, C., and Tuskan, G. A. 2011 Discovery and annotation of small proteins using genomics, proteomics, and computational approaches. Genome Res. 21:634-641.

Yeats, T. H., and Rose, J. K. C. 2008. The biochemistry and biology of extracellular plant lipid-transfer proteins (LTPs). Protein Sci. 17: 191-198.

Zhang, Y. 2008. I-TASSER server for protein 3D structure prediction. BMC Bioinformatics 9:40.

Zhang, Y.-Z., and Shen, H.-B. 2017. Signal-3L 2.0: A hierarchical mixture model for enhancing protein signal peptide prediction by incorporating residue-domain cross-level features. J. Chem. Inf. Model. 57:988-999.

Zhou, F., Zhang, Z., Gregersen, P. L., Mikkelsen, J. D., de Neergaard, E., Collinge, D. B., and Thordal-Christensen, H. 1998. Molecular characterization of the oxalate oxidase involved in the response of barley to the powdery mildew fungus. Plant Physiol. 117:33-41.

Zuccaro, A., Lahrmann, U., and Langen, G. 2014. Broad compatibility in fungal root symbioses. Curr. Opin. Plant Biol. 20:135-145. 